Two- di mensi onal beam emi ssi on spect r oscopy for hydr ogen i sot ope negat i ve neut $r$ al beam i $n$ Large Hel i cal Devi ce

\begin{tabular}{|l|l|}
\hline $\begin{array}{l}\text { j our nal or } \\
\text { publ i cat i on } \mathrm{t} \text { i t l e }\end{array}$ & $\mathrm{Pl}$ asma Physi cs and Cont rol I ed Fusi on \\
\hline vol une & 62 \\
\hline number & 12 \\
\hline page r ange & 125011 \\
\hline year & $2020-10-28$ \\
\hline URL & ht t p: //hdl . handl e. net /10655/00012584 \\
\hline
\end{tabular}




\title{
Two-dimensional beam emission spectroscopy for hydrogen isotope negative neutral beam in Large Helical Device
}

\author{
T. Kobayashi ${ }^{1,2}$, M. Yoshinuma ${ }^{1}$, and K. Ida ${ }^{1}$ \\ ${ }^{1}$ National Institute for Fusion Science, National Institutes of Natural Sciences, Toki 509-5292, \\ Japan \\ 2 The Graduate University for Advanced Studies, SOKENDAI, Toki 509-5292, Japan \\ E-mail: kobayashi.tatsuya@nifs.ac.jp
}

\begin{abstract}
A new beam emission spectroscopy (BES) system that has improved lines of sight is installed in LHD, and routine measurement has been started in the 21st LHD experiment campaign in 2019-2020. The new system is optimized for hydrogen isotope experiments by equipping a rotatable large-diameter interference filter to be compatible with either the hydrogen or the deuterium beam emission component. An avalanche photo diode detector array having 8 $\times 8$ pixels is used for obtaining a radial-vertical image of electron density fluctuation covering the mid-radius to the plasma periphery. Spatial resolution and wavenumber cutoff are derived from equilibrium reconstruction and plasma kinetic profiles. Obtained fluctuation data is presented for a low field high beta discharge. The spatiotemporal structure of the fluctuations is clearly shown by Fourier correlation analyses.
\end{abstract}

\section{Introduction}

Cross field anomalous transport in magnetically confined plasmas is one of the key issues in fusion development [1]. Multi-scale fluctuation interaction and consequent 
flow structure formation has been pointed out to be essential in determining turbulence energy redistribution in the wavenumber space for more than a decade [2]. To clarify underlying physics of the cross field anomalous transport and the nonlinear structure formation, direct measurement of turbulent fluctuation is mandatory.

Beam emission spectroscopy (BES) is one of the active local density fluctuation diagnostics installed in many different plasma fusion devices including tokamaks $[3,4,5,6,7,8,9,10,11,12,13]$ and stellarators $[14,15,16,17,18,19]$. Owing to its easily accessible range of visible light wavelength with commercial optics and applicability of commonly equipped heating neutral beams as the probing beam, BES systems are successfully operated in many different situations. In particular, multi-channel and multi-dimensional measurement system with good locality and high spatiotemporal resolution is universally composed for unveiling the multi-scale interaction of microscale turbulences and meso- and macro-scale plasma activities. One of the prototypical applications of BES systems is for determining the role of turbulence in the L-H transition. See, e.g., [20]. Recently, a dynamic interaction among magnetic islands, heat pulses, and turbulences is clearly depicted by a BES system in DIII-D [21].

One of the difficulties in installing a BES system having good resolutions is to find lines of sight as parallel as possible to the magnetic field line. For realizing this requirement, a tangential view to torus with an acceptable brightness is mandatory. Finding lines of sight that satisfy those conditions is often challenging particularly in large superconducting devices having wide cryostat structures, such as the large helical 
device (LHD).

A new BES system has been installed in LHD, and routine measurement has been started in the 21st LHD experiment campaign in 2019-2020. Compared to previous BES systems in LHD [17, 18, 19], the radial resolution is improved approximately by $60 \%$, and the angle between the line of sight and the beam vector is reduced by $37 \%$ that enlarges the spectrum separation of the beam emission component from the background $H_{\alpha} / D_{\alpha}$ line. The angles between the lines of sight and the local magnetic field lines are $6.73 \pm 2.6$ degrees in average, which enables us to achieve the wavenumber resolution of $k_{\perp}<0.6 \mathrm{~cm}^{-1}$. The new system is optimized for hydrogen isotope experiments by equipping a rotatable large-diameter interference filter to be compatible with either the hydrogen or the deuterium beam emission component. An avalanche photo diode detector array having $8 \times 8$ pixels is used for obtaining a radial-vertical image covering the mid-radius to the plasma periphery. In this paper, we elaborate the diagnostic capability of the new BES system using obtained electron density fluctuation data.

The rest of the paper is organized as follows: Section 2 describes the hardware setup of the new BES system. Then, fluctuation measurement capability is discussed in section 3 by examining the spatial resolution and wavenumber cutoff. Section 4 presents the measurement results. The summary is given in section 5 . 


\section{Hardware setup}

In this section, we describe the hardware properties constructing the new BES system with emphasis on its capability for fluctuation measurement in hydrogen isotope experiment.

\subsection{System overview}

The system overview is illustrated in Fig. 1. The objective optics are installed in the LHD 6-O port, where a commercial camera lens (Mamiya $\mathrm{F} / 2.8, f=300 \mathrm{~mm}$ ) is utilized to obtain the magnification of $\sim 15$. Behind the camera lens, a $16 \times 16$ channel optical fiber array of a diameter of $0.8 \mathrm{~mm}$ is installed to obtain a $20 \times 20 \mathrm{~cm}^{2}$ radial-vertical $(R-Z)$ image of the plasma fluctuation. The two-dimensional fiber array is led to a large-diameter interference filter spectrometer (Bunkou-Keiki CLF-400) by optical fiber bundles through two junction panels inside and outside the LHD hall aiming for flexible fiber arrangement. The light image is converted to the parallel light by the entrance collimate camera lens $(\mathrm{F} / 2.8, f=400 \mathrm{~mm})$ for utilizing the single largediameter interference filter of a diameter of $\sim 160 \mathrm{~mm}$. The angle between the normal vector to the filter surface and the parallel light can be externally tunable for varying the transmitted central wavelength. The filtered light image is directly coupled on an $8 \times$ 8 pixels avalanche photo diode camera (Fusion Instrument Kft. APDCAM-10g) by the exit focal camera lens $(\mathrm{F} / 2.8, f=400 \mathrm{~mm})$, where 4 fiber images are coupled to one APD element having $1.6 \times 1.6 \mathrm{~mm}^{2}$ shape as shown in Fig. 2. In APDCAM-10g, two $4 \times 8$ 


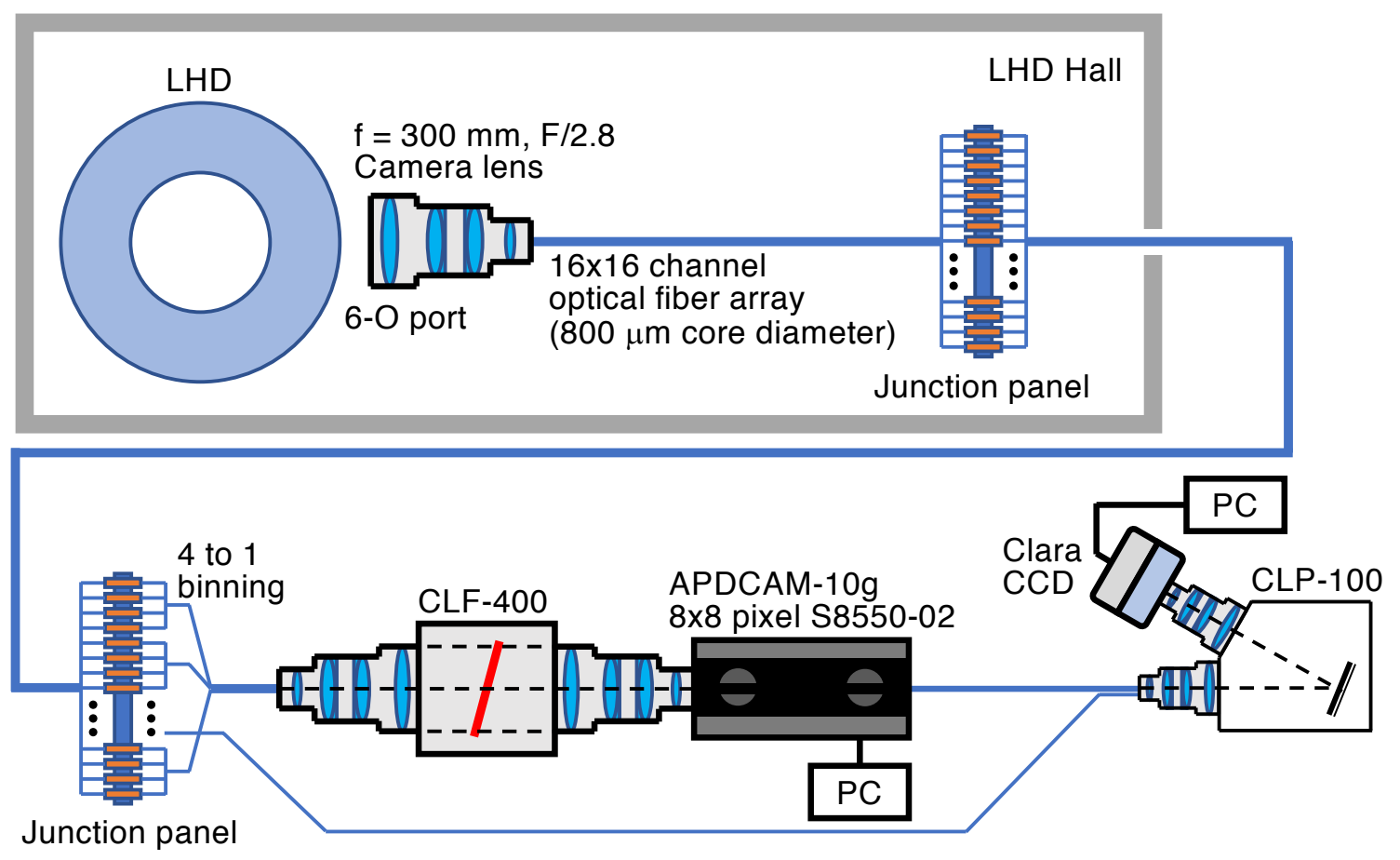

Figure 1. Schematic of the BES system in LHD.

pixels APD detector arrays (Hamamatsu S8550-02) are used. The frequency bandwidth is tuned to be $100 \mathrm{kHz}$ for obtaining a better signal to noise ratio in the low frequency range. Sampling time is set to be $200 \mathrm{kHz}$ so far. One of the advantages of APDCAM$10 \mathrm{~g}$ is that the exit fiber ports are equipped on the APD element surface. Some fiber spots are coupled to the exit fiber ports in order to monitor the transmitted spectrum by a conventional lens-grating spectrometer installed behind (Bunkou-Keiki CLP-100), which consists of a set of $\mathrm{F} / 2, f=100 \mathrm{~mm}$ camera lenses and an $1800 / \mathrm{mm}$ grating. In addition, the emission spectrum without filtering is always monitored. The detector of the lens-grating spectrometer is a Peltier-cooled charge-coupled device (CCD) camera (Clara, Andor) having a $1392 \times 1040$ pixel array with a $6.45 \times 6.45 \mu \mathrm{m}^{2}$ pixel size. 


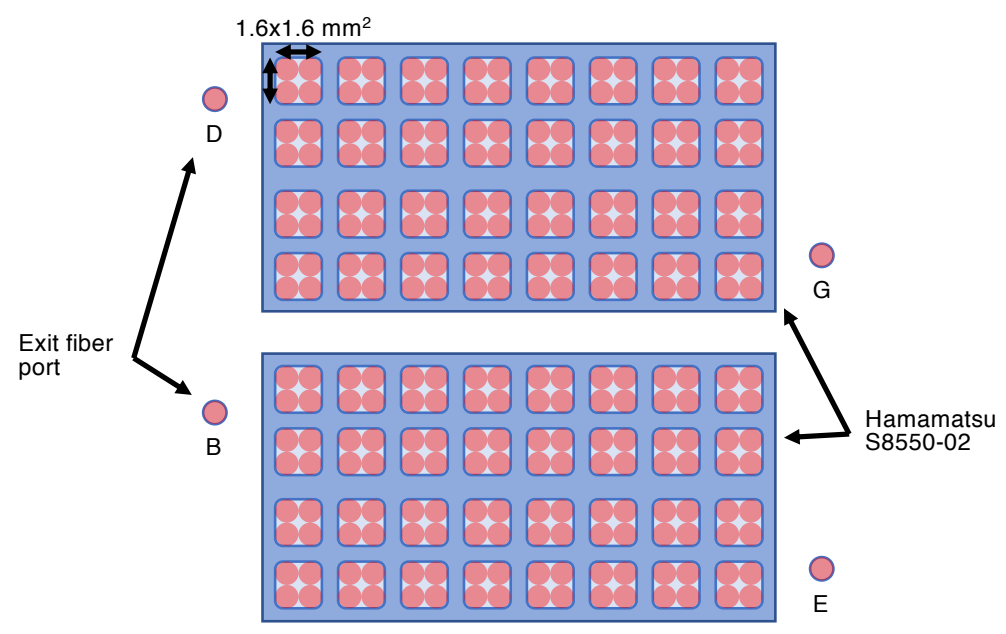

Figure 2. Schematic of the APD array arrangement.

\subsection{Viewing configuration and optical system}

Schematic view of the lines of sight is shown in Fig. 3 (a). One of the three neutral beam (NB) injection heating with the negative ion source (NB \#3) is used as the probe beam of the system. An in-vessel stainless steel mirror is installed to build lines of sight as parallel as possible to the local magnetic field line for obtaining a better wavenumber resolution. In order to protect the stainless steel mirror surface, a mechanical shutter system is also installed. The actuator of the mirror shutter is installed from the LHD 6-T port, where an air motor driving system with a rotary vacuum feedthrough and rotational shafts of totally $2.8 \mathrm{~m}$ long is utilized. The distance between the objective optics and the focal point is approximately $5 \mathrm{~m}$, and a single spot diameter at the focal point is $1.3 \mathrm{~cm}$. Angles between the lines of sight and the beam vectors are in the range of $150.6-153.6$ degrees. The neutral beam is injected with the beam energy of $120-180 \mathrm{keV}$ and the beam source of either hydrogen or deuterium, anticipating 

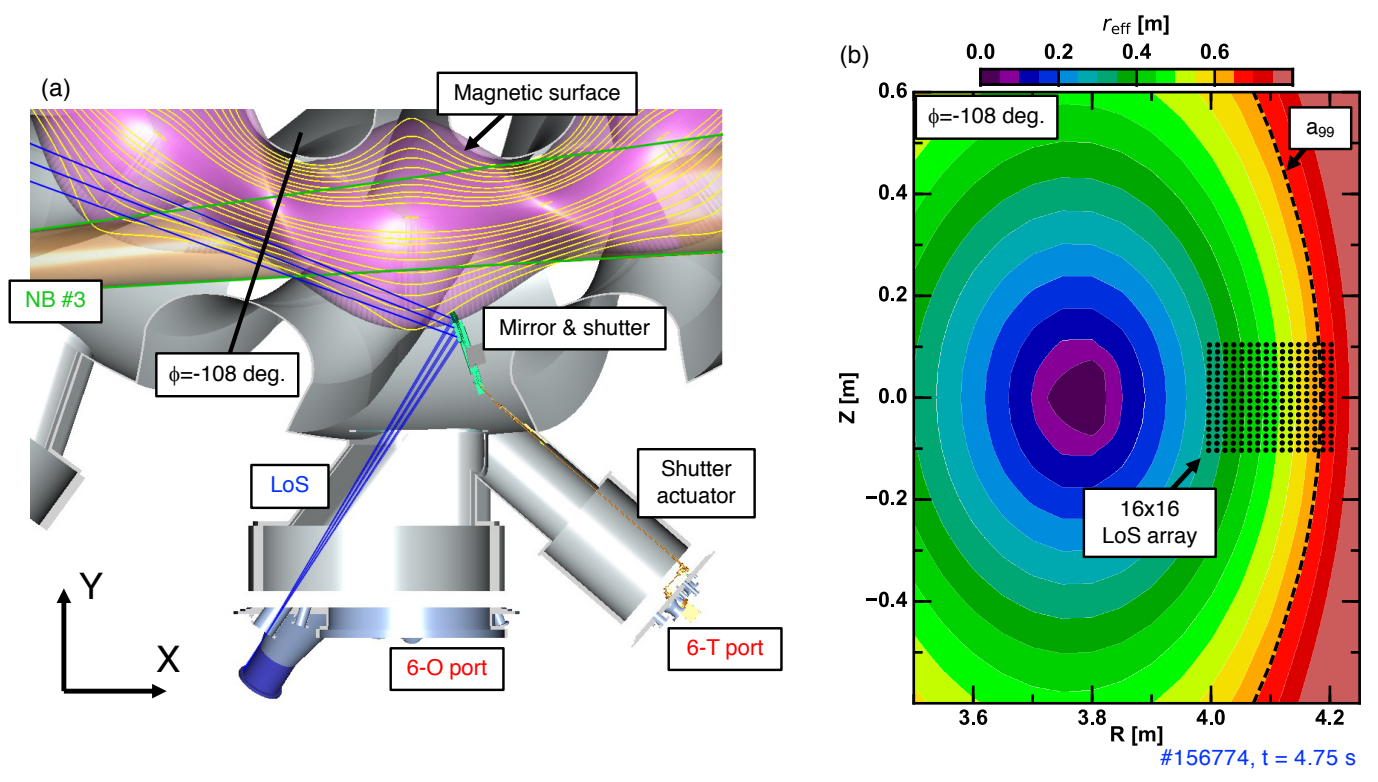

Figure 3. Schematic of the lines of sight (LoS) (a) on the top torus view and (b) on the poloidal cross section at $\phi=-108$ degrees overlaid with the effective minor radius.

the beam emission component having Doppler blue shifts of $6.46-11.51 \mathrm{~nm}$ from the $H_{\alpha} / D_{\alpha}$ line.

The two-dimensional array shape of the lines of sight is designed on the poloidal cross section at the toroidal angle of $\phi=-108$ degrees. Figure 3 (b) shows the schematic of the lines of sight on the poloidal cross section at $\phi=-108$ degrees overlaid with the effective minor radius $r_{\text {eff }}$, the most frequently used minor radial coordinate in LHD obtained according to the plasma volume [22]. The plasma boundary is defined by the effective minor radius inside which $99 \%$ of the plasma stored energy is confined, $a_{99}$. It is found that the radial region roughly from the mid-radius to the edge plasma boundary is covered by the two-dimensional array. The major radial position and the vertical position of the lines of sight on the poloidal cross section at $\phi=-108$ degrees, $(R, Z)$, are used as the nominal coordinate of the measurement. Note that the actual 
measurement location is determined by the line averaged effective minor radius weighted by the beam density and the electron density as discussed below.

The lifetime of the excited beam atoms is approximately $6-7 \mathrm{~ns}$ in the LHD parameter range $[23,5]$. The excited beam atom can penetrate deeper into the plasma with the high beam velocity of $\sim 4 \times 10^{6} \mathrm{~m} / \mathrm{s}$. Because the beam is not injected directly in the radial direction but in the tangential direction, the mean radial traveling extent in the lifetime is less than $\sim 1.3 \mathrm{~cm}$.

\subsection{Rotatable filter spectrometer system}

Since the neutral beam injection with the negative ion source has no multiple charged ions, only the fundamental Doppler shifted component need be taken care of. To minimize contaminations of impurity emissions or bremsstrahlung emissions, it is beneficial to use a narrow bandpass interference filter for this situation. In LHD deuterium/hydrogen experiments, the ion source for the neutral beam operation is

preferentially chosen to match the plasma source gas. Therefore, the transmitted central wavelength must be tunable according to the neutral beam source. To meet this requirement, a rotatable filter spectrometer system is designed [24].

Figure 4 (a) shows the rotation angle dependence of the transmitted central wavelength. The full width at the half maximum (FWHM) of the transmittance spectrum is shown by shaded areas. Those data are obtained by a bench test [24]. The transmitted central wavelength from the exit fiber port B is more blue shifted 

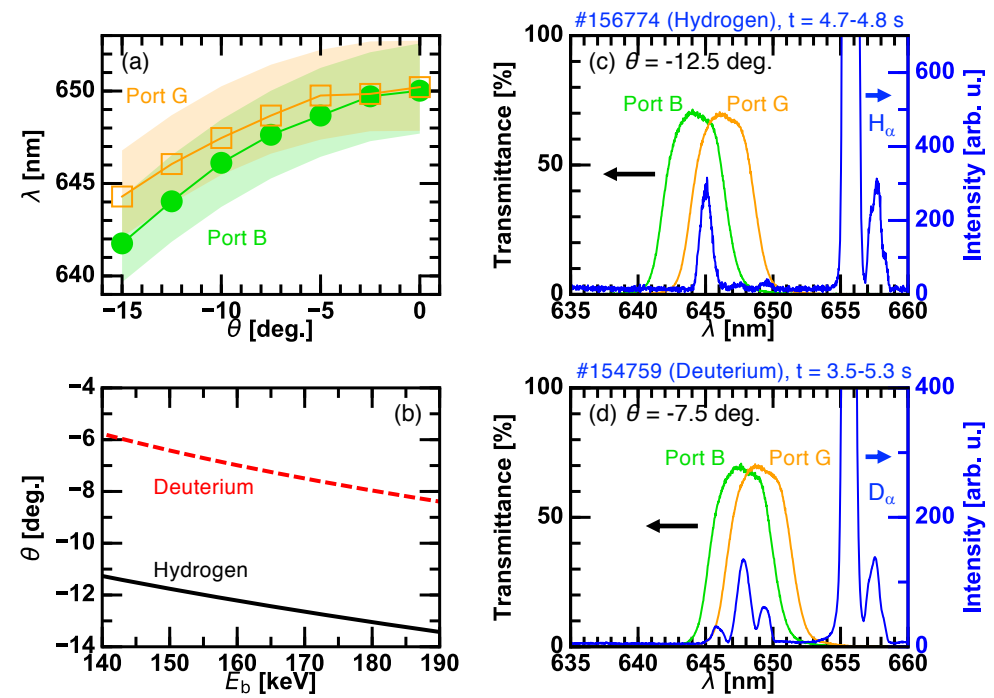

Figure 4. Specifications of the rotatable narrow bandpass filter spectrometer: (a) rotation angle $\theta$ dependence of the transmitted central wavelength with the full width at the half maximum (FWHM) of the transmittance spectrum shown by shaded areas for the exit fiber ports B and G, (b) rotation angle setting required for hydrogen and deuterium neutral beams as a function of the injection beam energy, and $(\mathrm{c}, \mathrm{d})$ transmission characteristics for different rotation angles $(\theta=-12.5 \mathrm{deg}$. and $\theta=-7.5 \mathrm{deg}$.) overlaid with typical beam emission spectra for hydrogen and deuterium neutral beams, respectively.

compared to that from the exit fiber port $\mathrm{G}$ because of a larger effective incident angle of the light to the filter which depends on the image position. Since the APD elements locate between those exit fiber ports, the transmitted wavelength of light focused on APD elements is a value between two wavelength curves in Fig. 4 (a). Necessary wavelength depends both on the neutral beam ion species and on the neutral beam energy. Therefore, rotation angle setting is given for each of those conditions [Fig. 4 (b)]. The most frequently used beam energy is $E_{\mathrm{b}} \sim 165 \mathrm{keV}$, where corresponding setting angles for the hydrogen beam and the deuterium beam are -12.5 degrees and 
-7.5 degrees, respectively. The transmittance spectra for those rotation angle settings are shown in Figs. 4 (c) and (d), overlaid with typical emission spectra around the beam emission components with hydrogen beam and deuterium beam, respectively. It is shown that the shapes of the transmittance spectra from port B and port $G$ with different rotation angle settings are almost identical, as is systematically demonstrated in [24]. The beam emission components locate between the transmittance spectra from port $B$ and port $G$. Note that detector columns at the port $G$ side (the right side columns on Fig. 2) suffer a reduction of signal intensity because the beam emission component lies at the edge of the transmission curve not at the flattop region with the rotation angle settings in Figs. 4 (c) and (d). To compensate this effect, we apply a signal calibration using a density ramp up discharge as discussed in subsection 4.2. In the case of the deuterium discharge [Fig. $4(\mathrm{~d})$ ], not only the beam emission component but also impurity emission lines (likely OV emissions from the edge region) exist in the transmitted range of wavelength, which worsens the locality of the BES signal. The plasma facing components were not well conditioned in that discharge, because it was immediately after an unplanned vacuum opening. In particular, approximately $40 \%$ of the transmitted light is estimated to be contaminated by those impurity emission lines according to the measured spectrum, therefore we have to abandon to use this data for analysis. An advantage of the new BES system is that the data quality is always checked by the monitor spectrometer. 


\section{Fluctuation measurement capability}

In this section, the fluctuation measurement capability of the new BES system is examined by calculating the radial resolution and the wavenumber cutoff from the reconstructed three-dimensional equilibrium and measured kinetic profiles for the hydrogen discharge \# 156774.

\subsection{Radial resolution}

The radial resolution of the BES lines of sight is analyzed on a simplified model illustrated in Fig. 5. The axis along the line of sight is defined as $z$, and local polar coordinates $\left(r_{\mathrm{F}}, \theta_{\mathrm{F}}\right)$ are defined on each poloidal cross section along the line of sight.

We consider variations in the neutral beam density $n_{\mathrm{b}}$ and the electron density $n_{\mathrm{e}}$ along the line of sight. In addition, an electron density variation on the poloidal cross section is taken into account, where the neutral beam density remains constant. The light emission intensity from the sampling volume is proportional to the volume integral of $n_{\mathrm{b}} n_{\mathrm{e}}$

$$
I=\int_{0}^{z} \int_{0}^{a_{\mathrm{F}}} \int_{0}^{2 \pi} n_{\mathrm{b}} n_{\mathrm{e}} r_{\mathrm{F}} d \theta_{\mathrm{F}} d r_{\mathrm{F}} d z
$$

where $a_{\mathrm{F}}$ is the local radius of the sampling volume cylinder. The electron density distribution in the sampling volume is approximated up to the first order terms as

$$
n_{\mathrm{e}}=\hat{n}_{\mathrm{e}}\left[1-r_{\mathrm{F}} L_{n_{\mathrm{e}}}^{-1}\left(\left.\cos \theta_{\mathrm{F}} \frac{\partial r_{\mathrm{eff}}}{\partial R}\right|_{r_{\mathrm{F}}=0}+\left.\sin \theta_{\mathrm{F}} \frac{\partial r_{\mathrm{eff}}}{\partial Z}\right|_{r_{\mathrm{F}}=0}\right)\right],
$$

where $L_{n_{\mathrm{e}}}^{-1} \equiv-\hat{n}_{\mathrm{e}}^{-1} \partial n_{\mathrm{e}} /\left.\partial r_{\mathrm{eff}}\right|_{r_{\mathrm{F}}=0}$ is the inverse density gradient length and $\hat{n}_{\mathrm{e}}=\left.n_{\mathrm{e}}\right|_{r_{\mathrm{F}}=0}$.

Since the variation in the electron density is treated as symmetric, the integrated value 


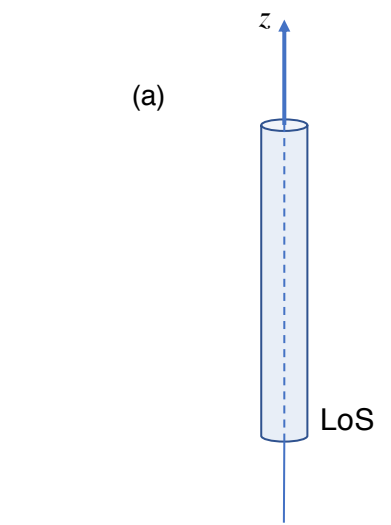

(c)

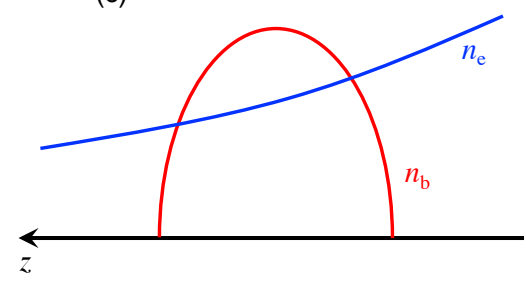

(b)

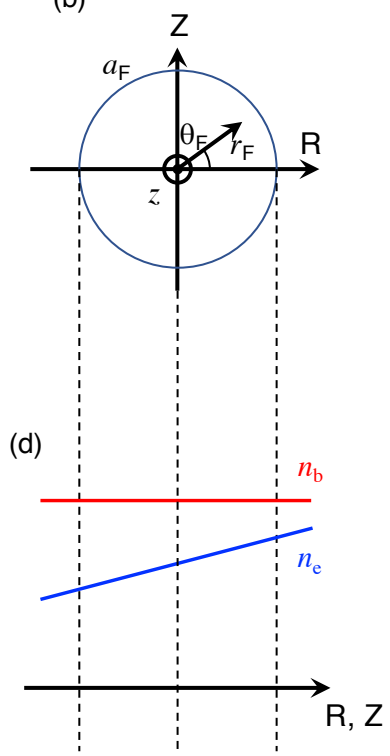

Figure 5. Schematic view of a sampling volume (a) along a line of sight (LoS) and (b) on the cross section perpendicular to the line of sight; models of the electron density profile and the neutral beam profile (c) along the line of sight and (d) on the cross section perpendicular to the line of sight.

is simply given as

$$
I=\pi a_{\mathrm{F}}^{2} \int_{0}^{z} n_{\mathrm{b}} \hat{n}_{\mathrm{e}} d z
$$

The mean radius of the sampling volume is given by the weighted integral of the effective minor radius $r_{\text {eff }}$ by $n_{\mathrm{b}} n_{\mathrm{e}}$ :

$$
\bar{r}_{\mathrm{eff}}=\frac{1}{I} \int_{0}^{z} \int_{0}^{a_{\mathrm{F}}} \int_{0}^{2 \pi} r_{\mathrm{eff}} n_{\mathrm{b}} n_{\mathrm{e}} r_{\mathrm{F}} d \theta_{\mathrm{F}} d r_{\mathrm{F}} d z
$$

The local effective minor radius in the sampling volume is given as

$$
r_{\mathrm{eff}}=\hat{r}_{\mathrm{eff}}+r_{\mathrm{F}}\left(\left.\cos \theta_{\mathrm{F}} \frac{\partial r_{\mathrm{eff}}}{\partial R}\right|_{r_{\mathrm{F}}=0}+\left.\sin \theta_{\mathrm{F}} \frac{\partial r_{\mathrm{eff}}}{\partial Z}\right|_{r_{\mathrm{F}}=0}\right) \text {, }
$$


where $\hat{r}_{\text {eff }}=\left.r_{\text {eff }}\right|_{r_{\mathrm{F}}=0}$. Now substituting Eq. (5) into Eq. (4) gives

$$
\bar{r}_{\mathrm{eff}}=\frac{\int_{0}^{z} \hat{r}_{\mathrm{eff}} n_{\mathrm{b}} \hat{n}_{\mathrm{e}} d z}{\int_{0}^{z} n_{\mathrm{b}} \hat{n}_{\mathrm{e}} d z}-\frac{1}{4} a_{\mathrm{F}}^{2} L_{n}^{-1}\left(\nabla r_{\mathrm{eff}}\right)^{2}
$$

where

$$
\left(\nabla r_{\mathrm{eff}}\right)^{2}=\left(\left.\frac{\partial r_{\mathrm{eff}}}{\partial R}\right|_{r_{\mathrm{F}}=0}\right)^{2}+\left(\left.\frac{\partial r_{\mathrm{eff}}}{\partial Z}\right|_{r_{\mathrm{F}}=0}\right)^{2}
$$

The first and second terms in Eq.(6) correspond to the mean effective minor radius along the line of sight and the correction term due to simultaneous variations of the effective minor radius and the electron density in the sampling volume, respectively.

Similarly, the deviation of the effective minor radius from its mean value, i.e., the radial resolution, is derived as

$$
\begin{aligned}
\left(\delta r_{\mathrm{eff}}\right)^{2} & =\frac{1}{I} \int_{0}^{z} \int_{0}^{a_{\mathrm{F}}} \int_{0}^{2 \pi}\left(r_{\mathrm{eff}}-\bar{r}_{\mathrm{eff}}\right)^{2} n_{\mathrm{b}} n_{\mathrm{e}} r_{\mathrm{F}} d \theta_{\mathrm{F}} d r_{\mathrm{F}} d z \\
& =\frac{\int_{0}^{z}\left(\hat{r}_{\mathrm{eff}}-\bar{r}_{\mathrm{eff}}\right)^{2} n_{\mathrm{b}} \hat{n}_{\mathrm{e}} d z}{\int_{0}^{z} n_{\mathrm{b}} \hat{n}_{\mathrm{e}} d z}+\frac{1}{4} a_{\mathrm{F}}^{2}\left(\nabla r_{\mathrm{eff}}\right)^{2}-\frac{1}{8} a_{\mathrm{F}}^{4} L_{n_{\mathrm{e}}}^{-2}\left(\nabla r_{\mathrm{eff}}\right)^{4} .
\end{aligned}
$$

The first and second terms reflect variations in the effective minor radius along the line of sight and perpendicular to the line of sight, respectively. The third term is the effective sampling volume shrinking due to the electron density variation.

Since the gradient length of the electron density and the scale length of the effective minor radius are much longer than the sampling volume radius, the correction terms in Eqs. (6) and (8) are negligibly small for all the lines of sight even in the peripheral density gradient region. Therefore, those terms are neglected in the rest of this paper. Calculations of the measurement position and the radial resolution are demonstrated using the actual discharge data. Figure 6 (a) shows the radial profiles of 

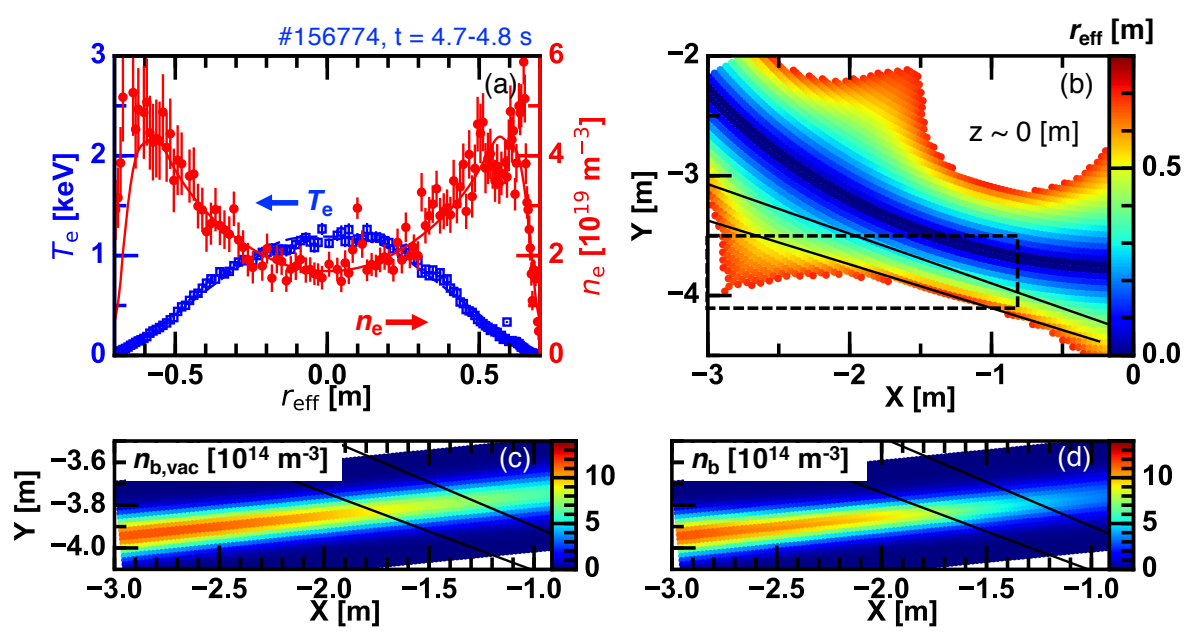

Figure 6. (a) Radial profiles of the electron density and the electron temperature measured by the Thomson scattering system, (b) reconstructed effective minor radius on the midplane, (c) vacuum neutral beam density profile, and (d) neutral beam density profile attenuated by plasma. Thin lines in (b-d) show the leftmost and the rightmost lines of sight passing through slightly above the midplane on the poloidal cross section at $\phi=-108 \mathrm{deg}$.

the electron density and the electron temperature determined by a Thomson scattering system [25]. In this discharge, a significant hollow electron density profile is formed. Spatial distribution of the effective minor radius is displayed in Fig. 6 (b). The neutral beam injection profile in vacuum is given by a neutral beam simulation code as shown in Fig. 6 (c). The plasma-beam interaction is calculated accounting for the beam stopping cross section depending on the beam energy, the electron density, the electron temperature, and the impurity contents [26]. The obtained neutral beam attenuation profile is shown in Fig. 6 (d).

Figure 7 shows distributions of the effective minor radius, the electron density, and the neutral beam density along the lines of sight with slightly above the midplane and 


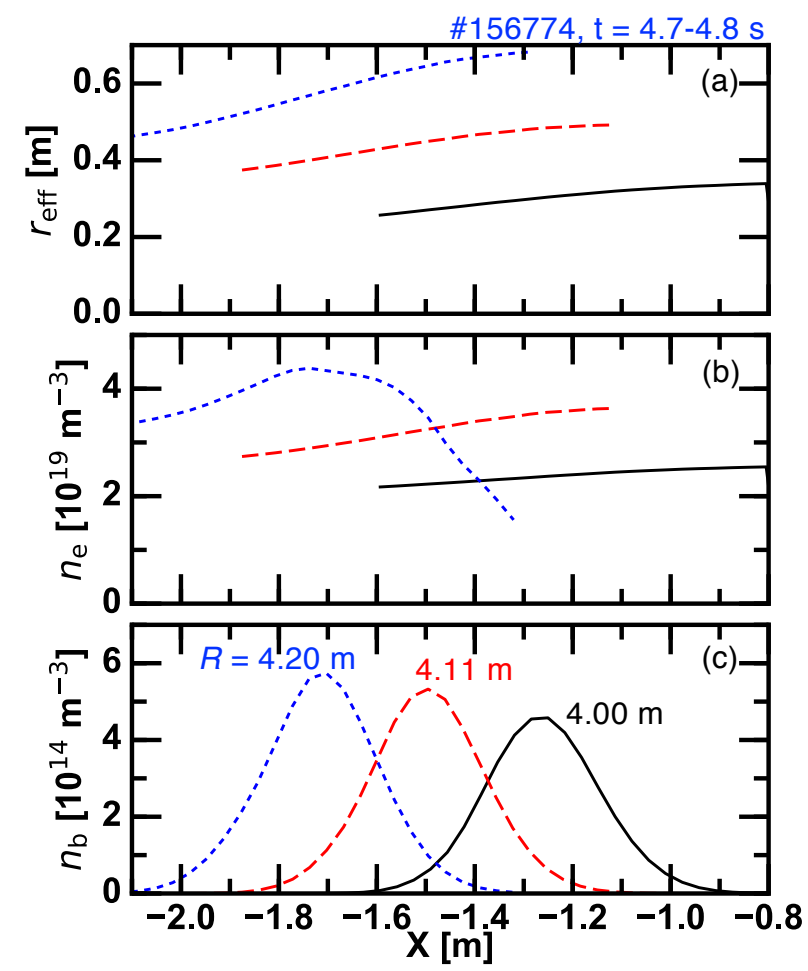

Figure 7. Distributions of (a) the effective minor radius, (b) the electron density, and (c) the neutral beam density along the lines of sight passing through slightly above the midplane and $R=4.00,4.11$, and $4.20 \mathrm{~m}$ on the poloidal cross section at $\phi=-108 \mathrm{deg}$.

$R=4.00,4.11$, and $4.20 \mathrm{~m}$ in the nominal coordinates. All three cases show similar beam density profile shapes with shifted central positions. As the nominal radial position moves outward, the effective minor radius increases and its deviation along the line of sight becomes larger. Variation in the electron density is particularly substantial at the edge line of sight.

Substituting obtained values to Eqs. (6) and (8) provides the mean effective minor radius and the radial resolution for each line of sight. They are exhibited in Figs. 8 (a) and (b) as a function of the nominal coordinates $(R, Z)$. The mean effective minor radius is larger at lines of sight having larger nominal $R$, but slightly changes in the 


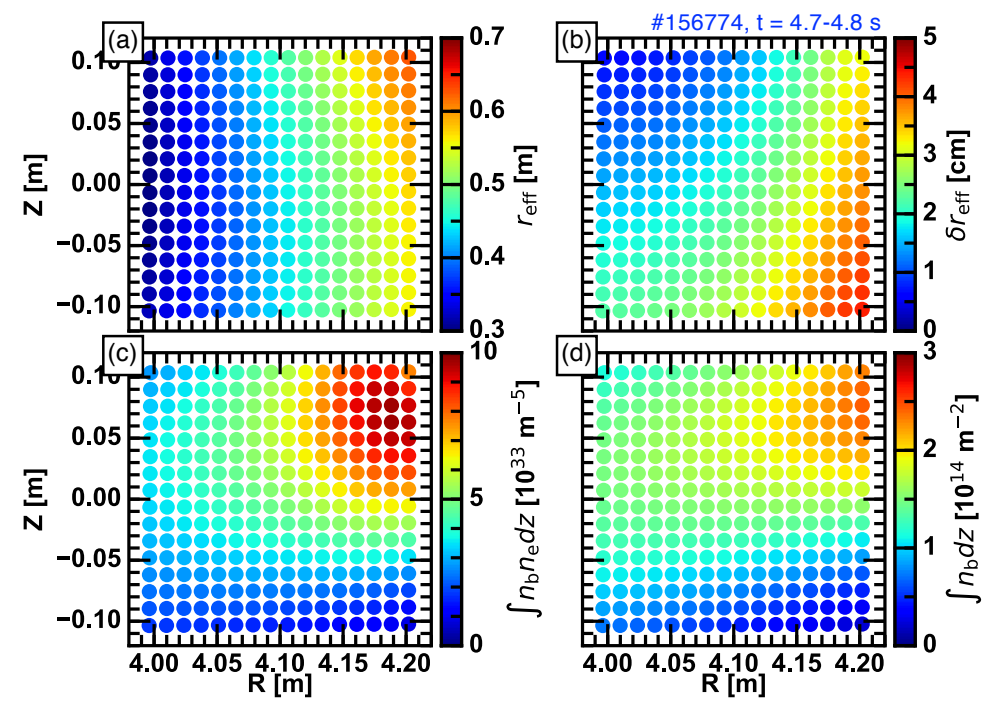

Figure 8. Distributions of (a) the mean effective minor radius, (b) the deviation of the effective minor radius, line integrations of (c) the neutral beam density multiplied by the electron density, and (d) the neutral beam density for all lines of sight plotted in the nominal coordinates.

$Z$ direction. The radial resolution is mostly less than $3 \mathrm{~cm}$, and worsens at the outer bottom lines of sight. Integrals of $n_{\mathrm{b}} n_{\mathrm{e}}$ and of $n_{\mathrm{b}}$ along the lines of sight are shown in Figs. 8 (c) and (d). Large signal intensities are expected in the outer top lines of sight.

\subsection{Fiber binning}

To increase signal intensity, lights from four sampling volumes are binned and coupled to one APD pixel, which would worsen the radial resolution. The mean effective minor radius with the fiber binning, $\bar{r}_{\text {eff,bin, }}$, is given as

$$
\begin{aligned}
\bar{r}_{\mathrm{eff}, \mathrm{bin}} & =\frac{1}{\sum_{j=1}^{N} I_{j}} \sum_{j=1}^{N} \int_{0}^{z} \int_{0}^{a_{\mathrm{F}}} \int_{0}^{2 \pi} r_{\mathrm{eff}, j} n_{\mathrm{b}, j} n_{\mathrm{e}, j} r_{\mathrm{F}} d \theta_{\mathrm{F}} d r_{\mathrm{F}} d z \\
& =\frac{\sum_{j=1}^{N} \int_{0}^{z} \hat{r}_{\mathrm{eff}, j} n_{\mathrm{b}, j} \hat{n}_{\mathrm{e}, j} d z}{\sum_{j=1}^{N} \int_{0}^{z} n_{\mathrm{b}, j} \hat{n}_{\mathrm{e}, j} d z}
\end{aligned}
$$


where the suffix $j$ denotes the fiber numbering for an APD pixel and $N=4$ is the number of the fiber binning. The mean deviation of the effective minor radius from $\bar{r}_{\text {eff,bin }}$ is given as

$$
\begin{aligned}
\left(\delta r_{\mathrm{eff}, \mathrm{bin}}\right)^{2} & =\frac{1}{\sum_{j=1}^{N} I_{j}} \sum_{j=1}^{N} \int_{0}^{z} \int_{0}^{a_{\mathrm{F}}} \int_{0}^{2 \pi}\left(r_{\mathrm{eff}, j}-\bar{r}_{\mathrm{eff}, \mathrm{bin}}\right)^{2} n_{\mathrm{b}, j} n_{\mathrm{e}, j} r_{\mathrm{F}} d \theta_{\mathrm{F}} d r_{\mathrm{F}} d z \\
& =\frac{\sum_{j=1}^{N} \int_{0}^{z}\left(\hat{r}_{\mathrm{eff}, j}-\bar{r}_{\mathrm{eff}, j}\right)^{2} n_{\mathrm{b}, j} \hat{n}_{\mathrm{e}, j} d z}{\sum_{j=1}^{N} \int_{0}^{z} n_{\mathrm{b}, j} \hat{n}_{\mathrm{e}, j} d z}+\frac{1}{N} \sum_{j=1}^{N}\left(\bar{r}_{\mathrm{eff}, j}-\bar{r}_{\mathrm{eff}, \mathrm{bin}}\right)^{2} .
\end{aligned}
$$

The first term shows the averaged radial deviation in all lines of sight on the APD pixel of interest, and the second term corresponds to deviation of the mean effective minor radii

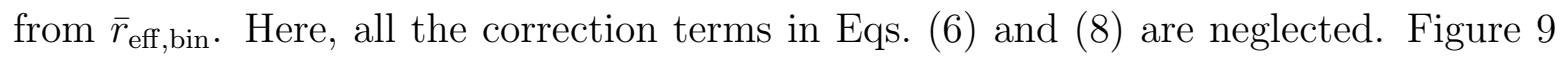
shows the mean effective minor radius and the radial resolution under the fiber binning for each detector channel plotted by the corresponding mean nominal coordinates. Here, symbols at the fifth row and the fifth column, the sixth row and the fourth column, and the sixth row and the fifth column from the top left corner are not aligned with the regular spacings. Those points are composed by the 3 fibers-to- 1 detector binning, and the remaining fiber channels are injected to exit fiber ports $B$ and $G$ and directly to the monitor spectrometer. The effect of the fiber binning is particularly clear in the radial resolution plot at the top inner positions, where the radial resolution before the fiber binning was finest. While, the radial resolution remains almost unchanged at the positions where $\delta r_{\text {eff }}$ before the fiber binning was larger than the sampling volume radius $a_{\mathrm{F}}$ of $0.65 \mathrm{~cm}$. 


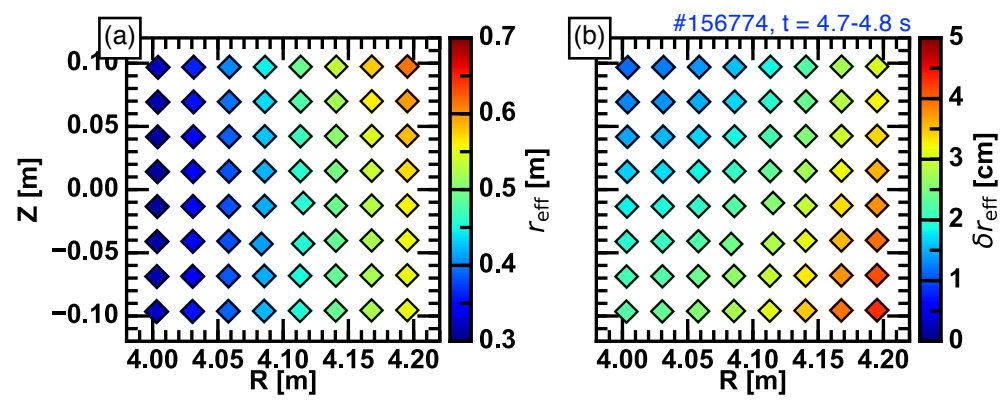

Figure 9. Distributions of (a) the mean effective minor radius and (b) the deviation of the effective minor radius under the 4 spots-to- 1 detector binning plotted in the mean nominal coordinates.
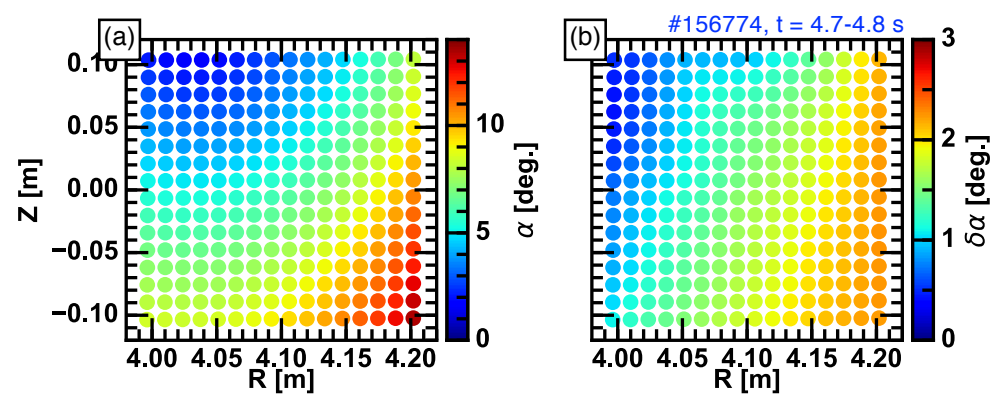

Figure 10. Distributions of (a) the angle between the line of sight and the magnetic field line and (b) its deviation plotted in the nominal coordinates.

\subsection{Angle between line of sight and magnetic field line}

The angle between the line of sight and the magnetic field line and its deviation on the line of sight, denoted as $\alpha$ and $\delta \alpha$, respectively, are calculated in the same ways to the effective minor radius. Distributions of $\alpha$ and $\delta \alpha$ are shown in Figs. 10 (a) and (b), respectively. Most of the lines of sight have $\alpha<8$ degrees with small deviations of $\delta \alpha<2$ degrees. Those angles determine the wavenumber cutoff as discussed below. 


\subsection{Wavenumber cutoff}

Volume integral of a temporally and spatially varying electron density in the sampling volume acts as a spatial lowpass filter, causing a fluctuation amplitude reduction in the BES signal. The amplitude reduction rate trivially depends on the fluctuation spatial structure. Here, the hardware wavenumber cutoff of the system is derived. The situation that is considered is illustrated in Fig. 11. A plane wave of the electron density having the parallel and perpendicular wavenumbers to the magnetic field direction, $k_{\|}$and $k_{\perp}$, is given as the test wave. Using the angle between the line of sight and the magnetic field line to be $\alpha$, the wavenumber components parallel and perpendicular to the line of sight, $k_{z}$ and $k_{x}$, are given as

$$
\left[\begin{array}{l}
k_{x} \\
k_{z}
\end{array}\right]=\left[\begin{array}{cc}
\cos \alpha & -\sin \alpha \\
\sin \alpha & \cos \alpha
\end{array}\right]\left[\begin{array}{l}
k_{\perp} \\
k_{\|}
\end{array}\right],
$$

where the direction $x$ is taken to be the direction of the wave propagation perpendicular

to the line of sight. The fluctuation part of the BES signal intensity is proportional to the volume integral of the electron density multiplied by the beam density denoted as

$$
\begin{aligned}
\tilde{I}_{j}(t) & \propto \int_{0}^{z} \int_{0}^{a_{\mathrm{F}}} \int_{0}^{2 \pi} n_{\mathrm{b}}(z)\left|\tilde{n}_{e}\right| e^{-i \omega t+i k_{x} x+i k_{z} z} r_{\mathrm{F}} d \theta_{\mathrm{F}} d r_{\mathrm{F}} d z \\
& =\left|\tilde{n}_{e}\right| e^{-i \omega t} \int_{0}^{z} n_{\mathrm{b}}(z) e^{i k_{z} z} d z \int_{0}^{a} \int_{0}^{2 \pi} e^{i k_{x} x} r_{\mathrm{F}} d \theta_{\mathrm{F}} d r_{\mathrm{F}}
\end{aligned}
$$

where $\left|\tilde{n}_{e}\right|$ is the amplitude of the electron density fluctuation, $\omega$ is the intrinsic frequency

of the wave, and $i$ is the imaginary unit. Signal intensity with the fiber binning is then given as

$$
\tilde{I}_{\mathrm{BES}}(t)=\sum_{j=1}^{N} \tilde{I}_{j}(t)
$$




$$
\propto\left|\tilde{n}_{e}\right| e^{-i \omega t} \int_{0}^{z} n_{\mathrm{b}}(z) e^{i k_{z} z} d z\left[\sum_{j=1}^{N} \int_{0}^{a} \int_{0}^{2 \pi} e^{i k_{x} x_{j}} r_{\mathrm{F}, j} d \theta_{\mathrm{F}, j} d r_{\mathrm{F}, j}\right]
$$

where differences among the binned lines of sight in the $z$ direction are negligibly small and therefore are not taken into account for simplicity. The integral in the $z$ direction and the term in the square brackets in Eq. (13) are called the parallel integral and the perpendicular integral, respectively. They and their product are shown in Fig. 12 as a function of the perpendicular wavenumber and the angle between the line of sight and the magnetic field line. Here, the parallel wavenumber is given by the gyrokinetic ordering to be $k_{\|} \sim \rho_{*} k_{\perp}$, where $\rho_{*} \equiv \rho_{\mathrm{t}, \mathrm{i}} / a_{99}$ is the relative ion gyroradius and $\rho_{\mathrm{t}, \mathrm{i}}$ is the ion gyroradius. Reduction of the fluctuation amplitude due to the volume integral becomes significant when the wave phase substantially changes in the direction of interest. The amplitude cutoff due to the perpendicular integral is significant when the perpendicular wavelength is comparable to the binned sampling volume size at $k_{\perp} \sim 1 \mathrm{~cm}^{-1}$. This cutoff wavenumber in the perpendicular integral is almost independent on the line of sight angle $\alpha$.

When the line of sight is perfectly aligned on the magnetic field direction, $\alpha=0$, the parallel integral is very weak because of its small parallel wavenumber. However, once the line of sight angle becomes finite, the perpendicular phase variation affects the phase distribution along the line of sight through Eq. (11), which strongly limits the observable perpendicular wavenumber range. As a result, the amplitude cutoff due to the parallel integral show a strong $\alpha$ dependence as shown in Fig. 12 (b). The product of both integrals is shown in Fig. 12 (c). The perpendicular wavenumber that results in 


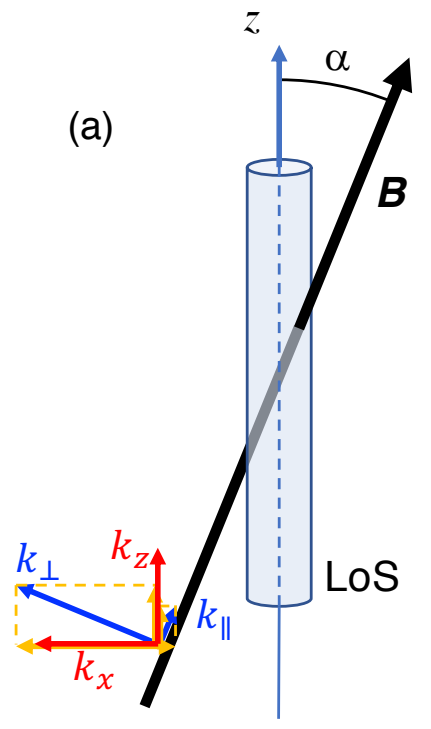

(b)

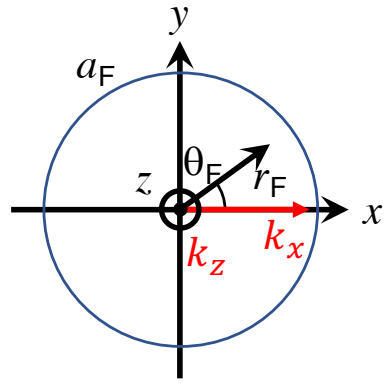

Figure 11. Schematic view of a sampling volume and a magnetic field line (a) along a line of sight (LoS) and (b) on the cross section perpendicular to the line of sight. Wavenumber vector components are also displayed.

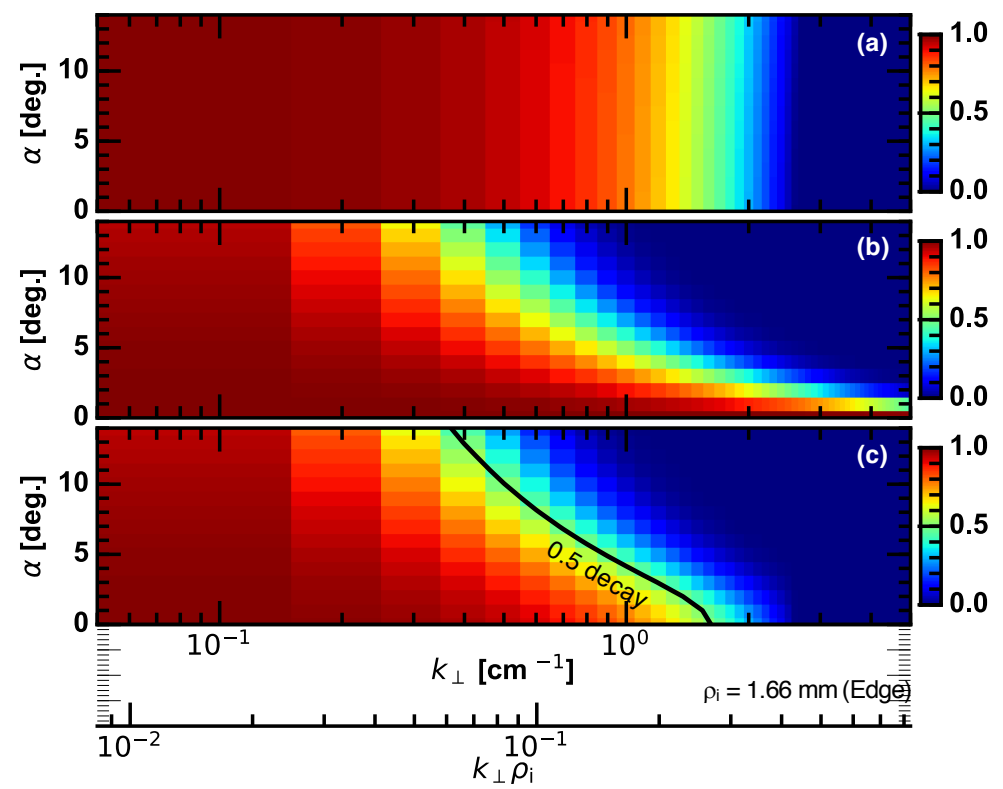

Figure 12. Fluctuation amplitude reductions as a function of the perpendicular wavenumber and the angle between the line of sight and the magnetic field line $\alpha$ by (a) the perpendicular integral, (b) the parallel integral, and (c) their product. A bold curve in (c) presents the wavenumber cutoff. 

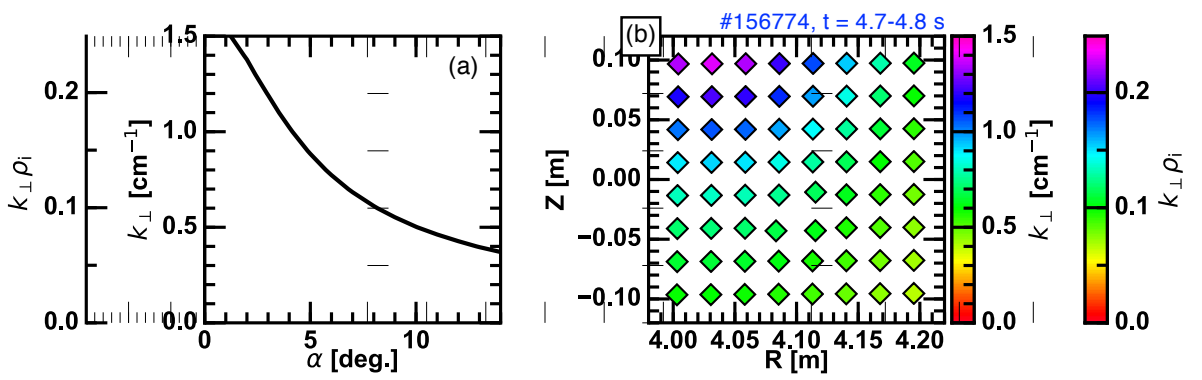

Figure 13. The wavenumber cutoff (a) plotted as a function of the angle between the line of sight and the magnetic field line and (b) plotted for all lines of sight in the nominal coordinates.

the half decay of the fluctuation amplitude, being referred to as the wavenumber cutoff, is overlaid by the black thick curve.

The wavenumber cutoff is plotted as a function of $\alpha$ in Fig. 13 (a). As shown above, $\alpha<8$ degrees holds for most of the lines of sight, which provides the wavenumber cutoff of $k_{\perp}>0.6 \mathrm{~cm}^{-1}$. The wavenumber cutoff is plotted for each binned line of sight in Fig. $13(\mathrm{~b})$.

Wavenumber coverage of the BES system is compared to other fluctuation diagnostics, the phase contrast imaging (PCI) [27], and the Doppler back scattering (DBS) [28] diagnostics in Fig. 14. The PCI and the DBS have lower boundaries in their wavenumber coverage, while the BES has an upper boundary. The BES complements the smaller wavenumber range that cannot be encompassed by the PCI and the DBS. The BES coverage is mostly out of the so-called turbulence range, typified by regimes of the ion temperature gradient (ITG) modes or the trapped electron modes (TEM), but is rather proper for MHD turbulences or long-wavelength fluctuations as shown in Fig. 14. The neutral beam with the negative ion source in LHD used as the probe beam 


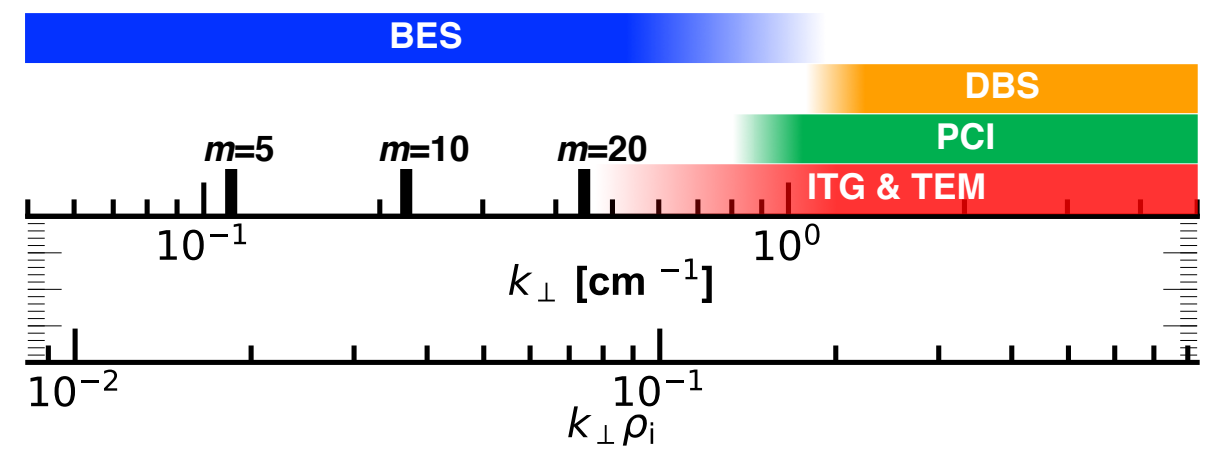

Figure 14. Observable wavenumber range of BES shown with covering ranges of the phase contrast imaging (PCI) diagnostics and the Doppler back scattering (DBS) diagnostic in LHD as well as typical wavenumber ranges for the ion temperature gradient (ITB) mode, the trapped electron mode (TEM), and coherent MHD modes with some poloidal mode numbers $m$.

of BES has an extremely low level of beam source oscillation, which helps to diagnose low frequency density fluctuations.

\section{Measurement results}

The new BES system was routinely operated from the 21st LHD experiment campaign in 2019-2020. One of the best results was obtained in an experiment aiming to clarify a confinement mode transition phenomena in a low magnetic field condition. A set of fluctuation analyses was applied to the data and basic properties were successfully drawn.

\subsection{Target discharge}

Figure 15 is the time evolution of the discharge parameters and the BES signals $I_{\mathrm{BES}}$ for the target shot \# 156774. Fueling from the gas puff and the neutral beams with positive 
ion source (NB \#4 and \#5) as well as the wall recycling is performed by deuterium gas, while the neutral beams with the negative ion source (NB \#1 to \#3) are operated with hydrogen gas. The deuterium content of the plasma is between $60 \%$ to $75 \%$. The confinement mode transition occurs at $t \sim 4 \mathrm{~s}$ indicated by an abrupt increase of the density ramp up pace. The discharge is performed in a low toroidal magnetic field condition with $B_{\mathrm{t}}=-1 \mathrm{~T}$ and an inward shifted magnetic axis configuration of $R_{\mathrm{ax}}=3.6 \mathrm{~m}$. An intrinsic error field is compensated by an external coil field. The probe beam (NB \#3) is injected from the beginning of the discharge and breaks down at $t \sim 5 \mathrm{~s}$. Throughout the discharge, the line averaged density ramps up from 1.5 to $4 \times 10^{19} \mathrm{~m}^{-3}$, which is useful for the BES signal calibration. The bottom panel shows the time evolution of the emission light spectrum. The beam emission component appears at $\lambda \sim 645 \mathrm{~nm}$ when both the plasma and the probe beam are present, and vanishes after the break down of the NB \#3. Impurity emissions are not distinguishably large due to intensive wall conditioning by the boronization. The BES signals from channels 2 (mid-radius) and 50 (edge) show corresponding time evolution. Note that even after the break down of the NB \#3, the BES signal intensities remain nonzero, possibly due to non beam emission components, including nonzero impurity emissions or the bremsstrahlung emission. 


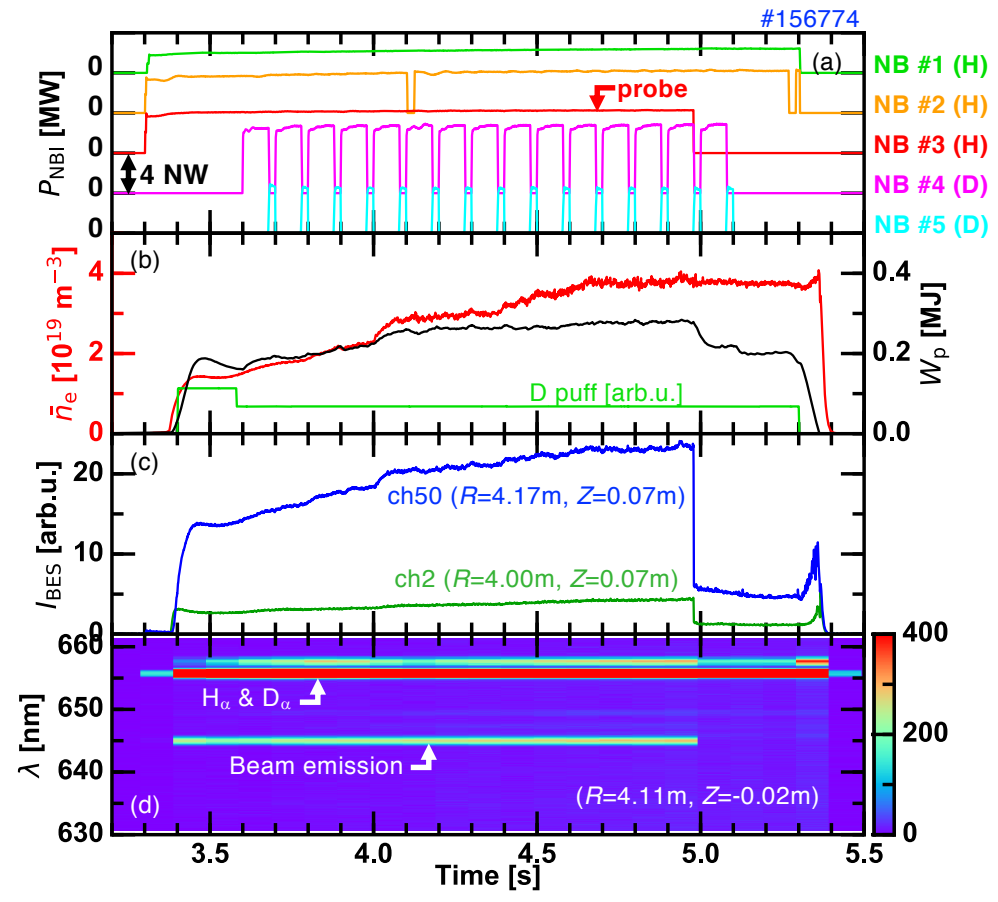

Figure 15. Time evolutions of (a) the NBI port through powers, (b) the line averaged density, the diamagnetic plasma stored energy, and the gas puff sequence, (c) the BES signal intensities of channels 2 and 50, and (d) the beam emission spectrum. The NB \# 3 is the probe beam of the BES system.

\subsection{Linearity between the BES intensity and the line averaged electron density}

The BES intensity is expected to be proportional to the integral of the electron density and the beam density along the line of sight, $\int n_{\mathrm{b}} n_{\mathrm{e}} d z$. Here, linearity between those two values are examined. Figure 16 compares the BES intensity and $\int n_{\mathrm{b}} n_{\mathrm{e}} d z$ for the BES channels 2 and 50. Calculation of $\int n_{\mathrm{b}} n_{\mathrm{e}} d z$ is presented in subsection 3.1. Values obtained by a moving averaging with a time window of $0.1 \mathrm{~s}$ from $3.5 \mathrm{~s}$ to $5.3 \mathrm{~s}$ are plotted. A value from $4.9 \mathrm{~s}$ to $5.0 \mathrm{~s}$ is excluded because the probe beam breaks down in the time period. The last three points with $\int n_{\mathrm{b}} n_{\mathrm{e}} d z=0$ correspond to values without the NB \#3 injection. Dashed lines in Fig. 16 present the linear fitting of the points when 

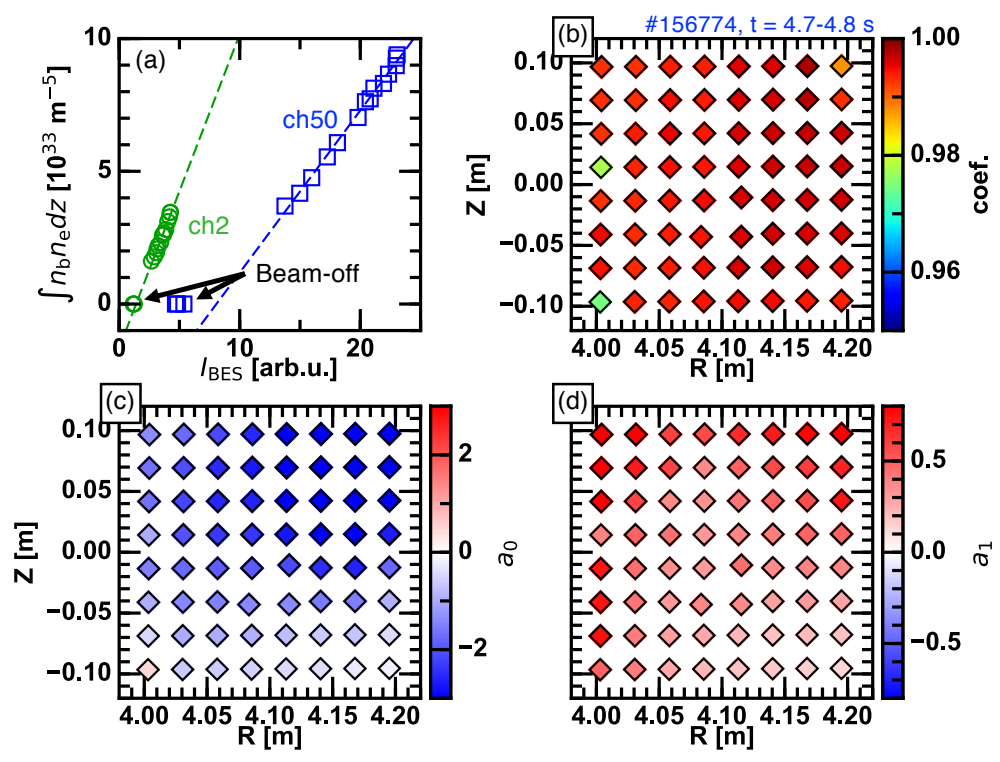

Figure 16. (a) Relation between the BES signal intensities and the neutral beam density multiplied by the electron density integrated on the lines of sight for the BES channels 2 (midradius) and 50 (edge), (b) the linear correlation coefficient, (c,d) the intersection $a_{0}$ and the slope $a_{1}$ of the linear fitting plotted in the nominal coordinates. Dashed lines in (a) are the linear fittings for symbols.

the probe beam is injected. Both of the BES channels show almost perfect linearities.

Points for the BES channel 50 without the NB \#3 injection do not meet the intercept of the fitting line. This implies that the BES signal has an offset possibly due to emissions caused by the probe beam injection. Correlation coefficients for the BES channels 2 and 50 are 0.995 and 0.997 , respectively. Very high linearity is a common feature in all channels as shown by Fig. 16 (b), where the correlation coefficients are plotted as a function of the mean nominal coordinates. Linear fitting coefficients, the intercept $a_{0}$ and the slope $a_{1}$, are also plotted in Figs. 16 (c) and (d).

Utilizing obtained linear fitting coefficients, the BES signals can be translated into 
the electron density. The line averaged electron density on the line of sight is given as

$$
n_{\mathrm{e}, \mathrm{LoS}}=\frac{\int n_{\mathrm{b}} n_{\mathrm{e}} d z}{\int n_{\mathrm{b}} d z}
$$

This value is a good approximation of the local electron density if $n_{\mathrm{e}}$ can be regarded as constant on the line of sight, i.e., $\int n_{\mathrm{b}} n_{\mathrm{e}} d z \sim n_{\mathrm{e}} \int n_{\mathrm{b}} d z$. The high linearity allows substitution of the numerator to the linear regression,

$$
n_{\mathrm{e}, \mathrm{BES}}=\frac{a_{0}+a_{1} I_{\mathrm{BES}}}{\int n_{\mathrm{b}} d z}
$$

The reconstructed electron density profiles are plotted with the Thomson measurement in Fig. 17 for different time periods. Here, fitted electron density profiles and their

uncertainties obtained based on a machine learning technique [29] are plotted. The horizontal errors show the radial resolution $\delta r_{\text {eff }}$, while the vertical errors indicate the electron density variation along the line of sight. The reconstructed profiles agree with the Thomson measurement except for the edge peak location, where the density variation along the line of sight is substantial as shown by Fig. 7 (b).

\subsection{Fluctuation analysis}

Fourier correlation analyses are applied to the BES data to obtain the basic spatiotemporal information of fluctuations. Figure 18 shows the time evolution of the squared cross coherence defined as

$$
\gamma^{2}(t, f)=\frac{\left|\left\langle S^{*} S_{\mathrm{ref}}\right\rangle_{t}\right|^{2}}{\left\langle S^{*} S\right\rangle_{t}^{2}\left\langle S_{\mathrm{ref}}^{*} S_{\mathrm{ref}}\right\rangle_{t}^{2}}
$$

where $S$ and $S_{\text {ref }}$ are the Fourier components of the BES signals at an arbitrary channel and at the reference channel, respectively. The Fourier transform is performed with the 


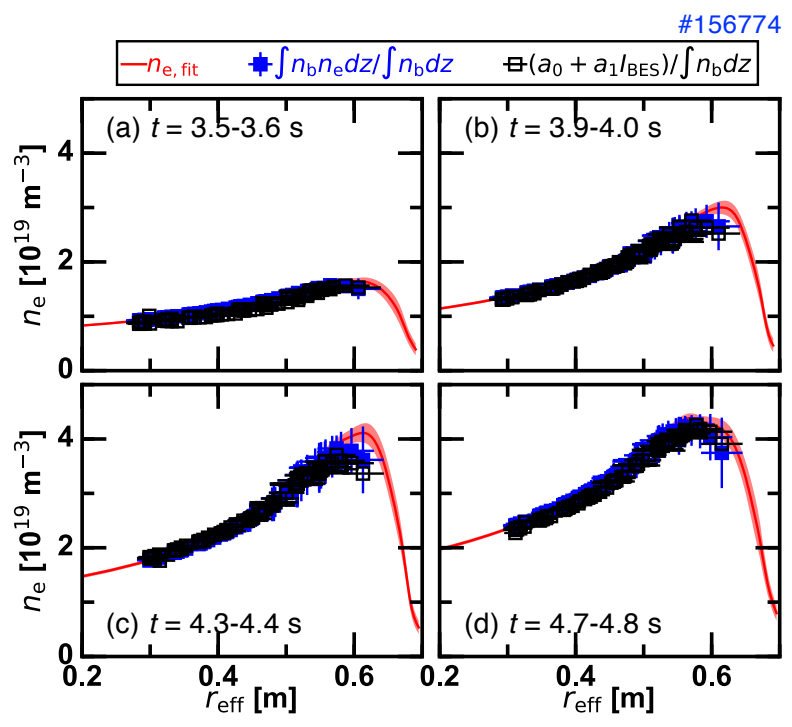

Figure 17. Fitted electron density profiles measured by Thomson scattering (red) and reconstructed electron density profiles by the beam attenuation calculation (blue) and by the BES measurement (black) for different time periods of (a) $t=3.5-3.6 \mathrm{~s}$, (b) $t=3.9-4.0 \mathrm{~s}$, (c) $t=4.3-4.4 \mathrm{~s}$, and (d) $t=4.7-4.8 \mathrm{~s}$.

$5 \mathrm{~ms}$ Turkey window and the $\Delta t=2.5 \mathrm{~ms}$ time interval. Moving time averaging is defined for an arbitrary quantity evolving in time $\Psi_{t}$ as $\langle\Psi\rangle_{t}=(2 N+1)^{-1} \sum_{i=-N}^{N} \Psi_{t+i \Delta t}$ with a factor of $N=7$. The reference channel is chosen to be the BES channel 49, at the top row and the seventh column from the top left corner of the two-dimensional array in Fig. 9 (a). The squared cross coherence is calculated for the radial channel array at the second row.

Far before the confinement mode transition occurring at $t \sim 4 \mathrm{~s}$, only a weak coherent mode appears at $f \sim 5 \mathrm{kHz}$. After the confinement mode transition, a variety of fluctuation activities are triggered. First, a low frequency coherent mode at $f \sim 1.4 \mathrm{kHz}$ is observed in all the BES channels. In addition, a broadband frequency 


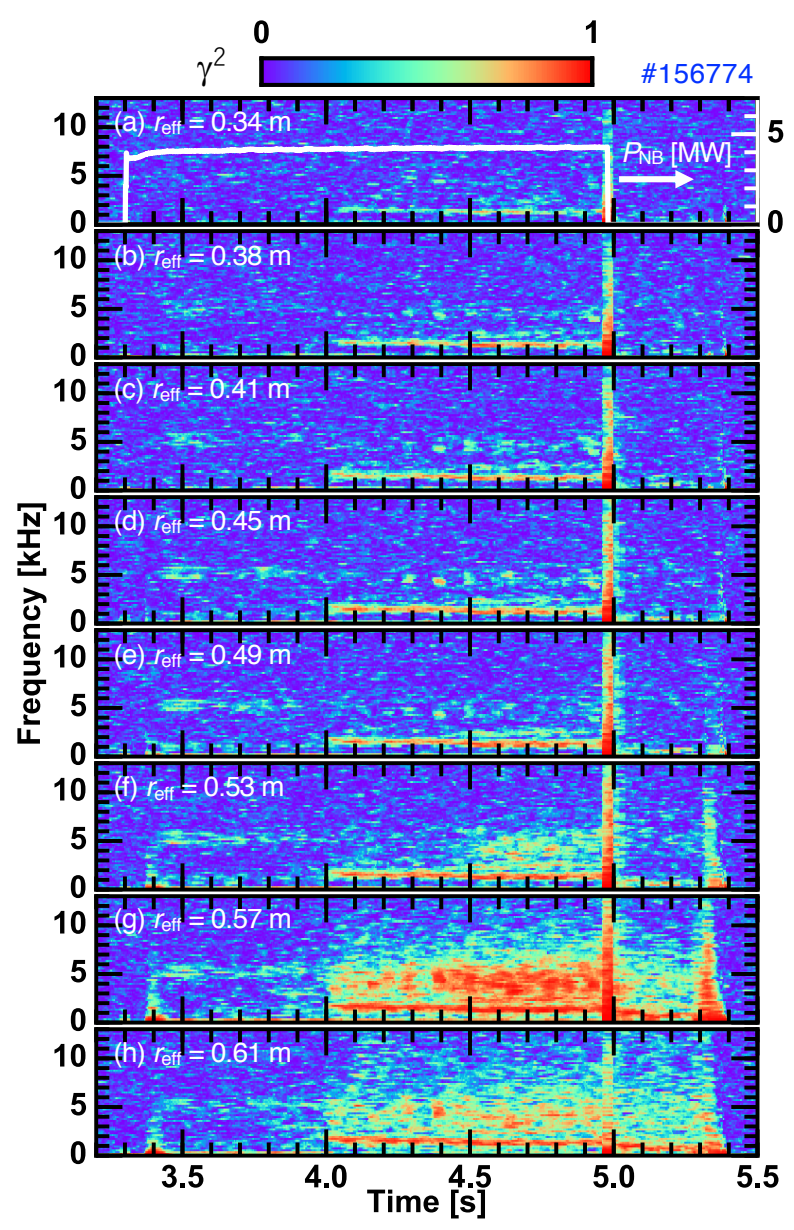

Figure 18. Time evolutions of the squared cross coherence at different radial positions of the second row of the two-dimensional array: (a) $r_{\text {eff }}=0.34 \mathrm{~m}$, (b) $r_{\text {eff }}=0.38 \mathrm{~m}$, (c) $r_{\text {eff }}=0.41 \mathrm{~m}$, (d) $r_{\text {eff }}=0.45 \mathrm{~m}$, (e) $r_{\text {eff }}=0.49 \mathrm{~m}$, (f) $r_{\text {eff }}=0.53 \mathrm{~m}$, (g) $r_{\text {eff }}=0.57 \mathrm{~m}$, and (h) $r_{\text {eff }}=0.61 \mathrm{~m}$. Reference signal is taken from the top row of the two-dimensional array at $r_{\text {eff }}=0.57 \mathrm{~m}$.

component in $f=2-10 \mathrm{kHz}$ is seen in the edge channels, whose coherence gradually increases in time and radial width expands in $t>4.5 \mathrm{~s}$. Shortly before the transition, both the low frequency and broadband fluctuation components seem to appear in some channels, although not as significant as those after the transition. Since the density is slowly ramped up before the transition as well, driving sources for those modes are possibly related to the density and/or the edge density gradient. Even after the break 
down of the probe beam NB \#3, a weak coherence remains in the edge channels, likely due to edge broadband emissions. A similar fluctuation spectrum is obtained from the magnetic probe array placed on the vacuum vessel wall, suggesting that the observed density fluctuations are caused by MHD activities.

Detailed discussion on the fluctuation properties is performed on time averaged spectrum in $t=4.70-4.97 \mathrm{~s}$, the latter phase of the improved confinement period. Figures 19 (a)-(c) show the power spectrum density, the squared cross coherence, and the cross phase, respectively, as a function of the frequency. Positive cross phase corresponds to the phase delay with respect to the reference channel. The spectral peaks are largest at the outermost radius, and gradually decays as the measurement position moves inward. The squared cross coherence is largest at the second outermost radius, i.e., the position closest to the reference channel at $r_{\text {eff }} \sim 0.57 \mathrm{~m}$. The squared cross coherences of the higher frequency peaks decay quicker than that of the lowest frequency peak at $f=1.4 \mathrm{kHz}$. The phase difference is nonzero in the entire frequency range, showing radial phase propagations.

Figures 19 (d)-(f) show the radial profiles of the power spectrum density, the squared cross coherence, and the cross phase, respectively, for spectral peaks at $f=1.4 \mathrm{kHz}$, $2.8 \mathrm{kHz}$, and $4.2 \mathrm{kHz}$. The peak values of the power spectrum density are larger than the photon noise level. High squared cross coherence of the $f=1.4 \mathrm{kHz}$ peak prevails over the observable radial range, while the coherent fluctuations of higher frequencies are mostly localized at the edge region. Smooth outward propagation of the fluctuation 


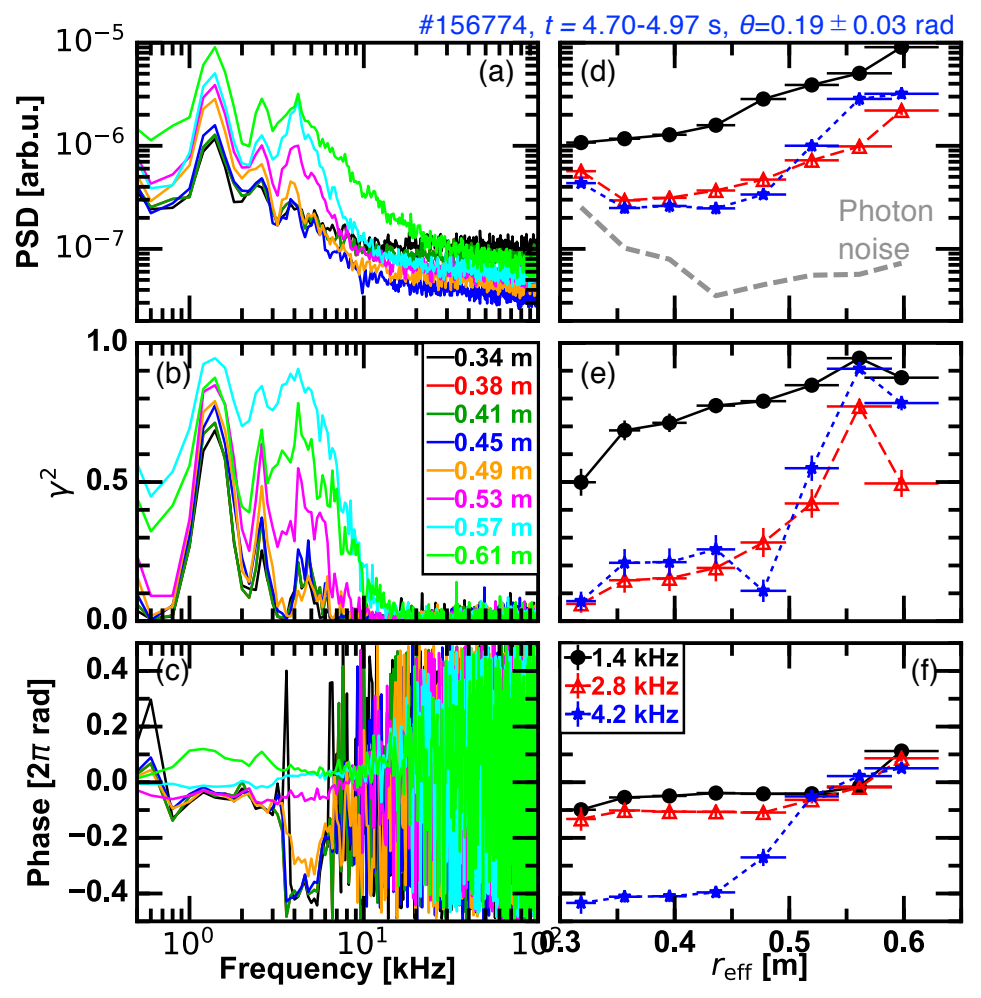

Figure 19. Frequency dependences of (a) the power spectrum densities, (b) the squared cross coherences, and (c) the cross phases at different radial positions and (d-f) their radial profiles for spectral peaks at $f=1.4 \mathrm{kHz}, 2.8 \mathrm{kHz}$, and $4.2 \mathrm{kHz}$.

phase is seen for all three frequency peaks, while the phase propagation velocity differs from each other. Since no discontinuous phase inversion is seen, observed radial structures may not be the so-called common modes due to the edge beam attenuation.

Poloidal structure of the fluctuation modes at $r_{\text {eff }} \sim 0.47$ (the seventh column of the two-dimensional array) is displayed in Fig. 20. The squared cross coherence and the cross phase are calculated with respect to the same reference signal of the BES channel 49, the top row and the seventh column of the two-dimensional array. Since the radial positions of the poloidal array are very close to each other, the power spectrum density levels are nearly identical as shown in Fig. 20 (a). The squared cross coherence decreases and 


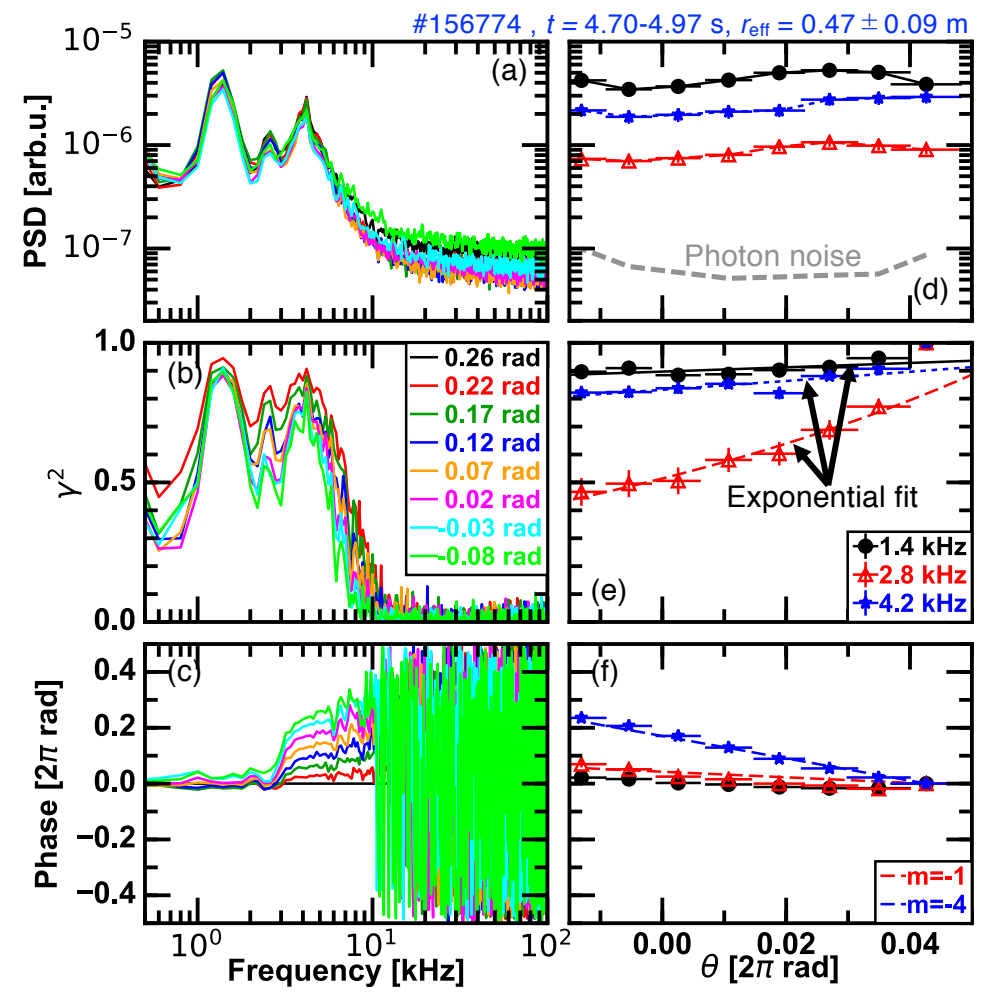

Figure 20. Frequency dependences of (a) the power spectrum densities, (b) the squared cross coherences, and (c) the cross phases at different poloidal angles and (d-f) their poloidal angle profiles at spectral peaks at $f=1.4 \mathrm{kHz}, 2.8 \mathrm{kHz}$, and $4.2 \mathrm{kHz}$.

the phase difference increases as the measurement position is apart from the reference channel, indicating preferential phase propagations in one direction [Figs. 20 (b) and (c)].

Detailed poloidal structure is presented in Figs. 20 (d)-(f) for spectral peaks at $f=1.4 \mathrm{kHz}, 2.8 \mathrm{kHz}$, and $4.2 \mathrm{kHz}$. The peak values of the power spectrum density are almost independent on the poloidal angle [Figs. 20 (d)]. The poloidal correlation length of the fluctuating mode can be derived from the squared cross coherence profile shown in Figs. 20 (e). By performing the exponential fitting to the squared cross coherence profiles except for the reference channel, the e-folding poloidal angles are obtained to 
be $7.35,0.573$, and 3.34 radians for fluctuations at $f=1.4 \mathrm{kHz}, 2.8 \mathrm{kHz}$, and $4.2 \mathrm{kHz}$, respectively. The fluctuations at $f=1.4 \mathrm{kHz}$ and $4.2 \mathrm{kHz}$ are global modes because the correlation remains high in the poloidal location isolated by $\pi$. Meanwhile, the fluctuation at $f=2.8 \mathrm{kHz}$ shows a much smaller correlation angle of $\sim \pi / 11$, presenting a local mode feature.

Similarly, performing the linear fitting to the phase profiles gives an estimation of the poloidal mode structure. Note that the general MHD mode structures in LHD are described in the Boozer coordinate system, therefore the fitting in the real coordinate does not necessarily provide an accurate determination of the poloidal mode number [30]. Nevertheless, it is worthwhile to obtain the approximated numbers in the real coordinate. The poloidal mode structure of the fluctuation at $f=4.2 \mathrm{kHz}$ is well approximated by $m=-4$ structure, where the negative number corresponds to the mode propagation in the electron diamagnetic direction. The fluctuation at $f=2.8 \mathrm{kHz}$ seems to have a poloidal mode number of $m \sim-1$, while for the fluctuation at $f=1.4 \mathrm{kHz}$, $-1<m<0$ is a possible estimation. A non integer $m$ is not realistic. Low $m$ modes only provides a small phase difference in different BES channels, therefore phase contaminations due to the radial variation of the BES channel position or the real coordinate-Boozer coordinate transformation may become meaningful. To obtain more plausible poloidal mode numbers using the BES data, further corrections required, which is one of the unresolved issues for the future.

Finally, two-dimensional images of the electron density fluctuation pattern are 


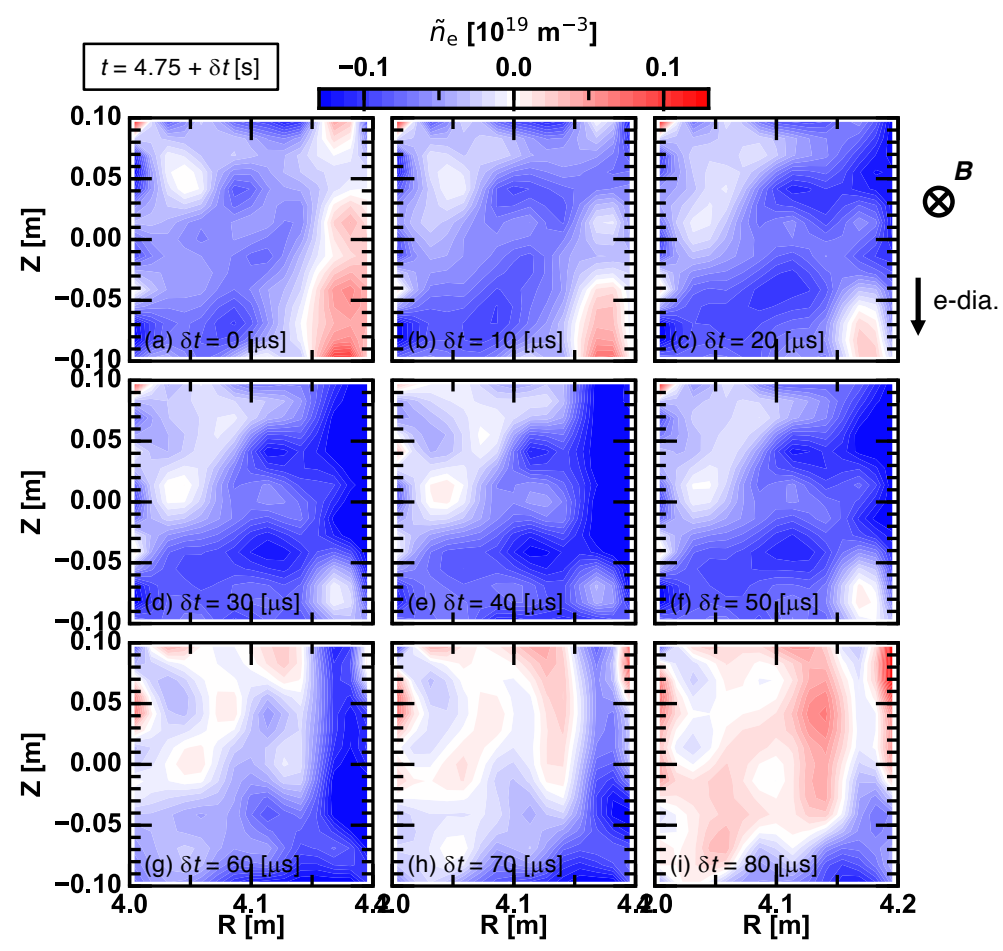

Figure 21. Time evolution of two-dimensional electron density fluctuation images in the frequency range of $2-10 \mathrm{kHz}$. Frames are separated by $10 \mu$ s each.

shown in Fig. 21. Signals from all BES channels are translated to the electron density by Eq. (15), and are processed by a digital bandpass filter with the transmitted frequency band of $f=2-10 \mathrm{kHz}$ for drawing out the turbulent nature. Snapshots are shown with a $10 \mu$ s interval. For the sake of better visualization, a spline interpolation is applied in both the $R$ and $Z$ directions. Although it is not very unambiguous, there are density clumps and holes propagating downward, i.e., the electron diamagnetic direction. The amplitude of the electron density fluctuation is $\sim 0.1 \times 10^{19} \mathrm{~m}^{-3}$ in average, corresponding to $\sim 2 \%$ of the equilibrium electron density. 


\section{Summary}

A new beam emission spectroscopy system, which has improved lines of sight and optimized rotatable interference filter spectrometer for hydrogen isotope experiments, was installed in LHD. An avalanche photo diode detector array having $8 \times 8$ pixels was used for obtaining a radial--vertical image of electron density fluctuation covering the mid-radius to the plasma periphery. Spatial resolution and wavenumber cutoff were calculated from equilibrium reconstruction and plasma kinetic profiles. Obtained fluctuation data was presented for a low field high beta discharge, and the spatiotemporal structure of the fluctuations was successfully shown.

The BES system introduced here is the only diagnostic system that can measure the local electron density without a lower wavenumber cutoff in LHD. Radial-vertical two-dimensional array is useful to image the low frequency electron density fluctuation mainly for global MHD activities. By operating the BES with turbulent density fluctuation measurement systems such as DBS or PCI, most of the wavenumber range can be covered, which is planned in the near future.

\section{Acknowledgments}

The authors acknowledge all the members of the LHD Experiment Group for their assistance. The authors also thank H. Nakano, Y. Fujiwara, M. Kisaki, K. Tanaka, T. Tokuzawa, M. Nakata, Y. Suzuki, C. Suzuki, M. Emoto, K. Yamasaki, C. Moon, T. Oishi, S. Kado, S. Kobayashi, G. R. McKee, Z. Yan, D. Dunai, W. Hu, V. Olevskaia, 
M. Osakabe, T. Morisaki, and S. Sakakibara for strong support and useful discussions. This work is partly supported by the National Institute for Fusion Science grants (ULHH033) and by the Grant-in-Aid for Scientific Research of JSPS (15H02336, 17K14898). 


\section{References}

[1] Ch P Ritz, R V Bravenec, P M Schoch, Roger D Bengtson, J A Boedo, J C Forster, Kenneth W Gentle, Y He, R L Hickok, Y J Kim, et al 1989 Phys. Rev. Lett. 621844

[2] P H Diamond, S I Itoh, K Itoh, and T S Hahm 2005 Plasma Phys. Control. Fusion 47 R35

[3] R J Fonck, P A Duperrex, and S F Paul 1990 Rev. Sci. Instrum. 61 3487-3495

[4] G R McKee, C Fenzi, R J Fonck, and M Jakubowski 2003 Rev. Sci. Instrum. 74 2014-2019

[5] M W Shafer, R J Fonck, G R McKee, and D J Schlossberg 2006 Rev. Sci. Instrum. $7710 \mathrm{~F} 110$

[6] G R McKee, R J Fonck, D K Gupta, D J Schlossberg, M W Shafer, R L Boivin, and W Solomon 2007 Plasma Fusion Res. 2 S1025-S1025

[7] A R Field, D Dunai, R Gaffka, Y-c Ghim, I Kiss, B Meszaros, T Krizsanoczi, S Shibaev, and S Zoletnik 2012 Rev. Sci. Instrum. 83013508

[8] Y-c Ghim, A R Field, D Dunai, S Zoletnik, L Bardóczi, A A Schekochihin, and MAST Team 2012 Plasma Phys. Control. Fusion 54095012

[9] D R Smith, R J Fonck, G R McKee, and D S Thompson 2012 Rev. Sci. Instrum. 8310 D502

[10] M Lampert, G Anda, A Czopf, G Erdei, D Guszejnov, Á Kovácsik, G I Pokol, D Réfy, Y U Nam, and S Zoletnik 2015 Rev. Sci. Instrum. 86073501

[11] M Lampert, S Zoletnik, J G Bak, Y U Nam, and KSTAR Team 2018 Phys. Plasmas 25042507

[12] Y Y Li, J Fu, B Lyu, X W Du, C Y Li, Y Zhang, X H Yin, Y Yu, Q P Wang, M von Hellermann, et al 2014 Rev. Sci. Instrum. 85 11E428

[13] R Ke, Y F Wu, G R McKee, Z Yan, K Jaehnig, M Xu, M Kriete, P Lu, T Wu, L A Morton, et al 2018 Rev. Sci. Instrum. 89 10D122

[14] S Kobayashi, S Kado, T Oishi, T Kagawa, S Ohshima, T Mizuuchi, K Nagasaki, S Yamamoto, H Okada, T Minami, et al 2010 Rev. Sci. Instrum. $8110 \mathrm{D} 726$

[15] S Kobayashi, S Ohshima, H Matsuda, X X Lu, D Kokubu, K Ida, T Kobayashi, M Yoshinuma, S Kado, T Oishi, et al 2016 Rev. Sci. Instrum. $8711 \mathrm{E} 519$ 
[16] T Oishi, S Tanaka, S Kado, M Yoshinuma, K Ida, S Okamura, and CHS group 2004 Rev. Sci. Instrum. 75 4118-4120

[17] S Kado, T Oishi, M Yoshinuma, and K Ida 2010 Rev. Sci. Instrum. 81 10D720

[18] T Oishi, S Kado, K Ida, M Yoshinuma, H Nakano, and K Yamazaki 2010 Rev. Sci. Instrum. 81 10D719

[19] M Ono, K Ida, M Yoshinuma, H Nakano, T Kobayashi, and C Moon 2016 Plasma Fusion Res. 11 $1402115-1402115$

[20] Z Yan, P Gohil, G R McKee, D Eldon, B Grierson, T Rhodes, and C C Petty 2017 Nucl. Fusion 57126015

[21] K Ida, T Kobayashi, M Ono, T E Evans, G R McKee, and M E Austin 2018 Physical review letters 120245001

[22] C Suzuki, K Ida, Y Suzuki, M Yoshida, M Emoto, and M Yokoyama 2012 Plasma Phys. Control. Fusion 55014016

[23] I H Hutchinson 2001 Plasma Phys. Control. Fusion 4471

[24] V Olevskaia, T Kobayashi, K Ida, M Yoshinuma, and W Hu 2019 Plasma Fusion Res. $141305118-$ 1305118

[25] I Yamada, K Narihara, H Funaba, T Minami, H Hayashi, T Kohmoto, and LHD Experiment Group 2010 Fusion Sci. Tech. 58 345-351

[26] K Ida, M Yoshinuma, T Kobayashi, Y Fujiwara, J Chen, I Murakami, M Kisaki, M Osakabe, and LHD Experiment Group 2019 Plasma Fusion Res. 141402079

[27] K Tanaka, C A Michael, L N Vyacheslavov, A L Sanin, K Kawahata, T Akiyama, T Tokuzawa, and S Okajima 2008 Rev. Sci. Instrum. 79 10E702

[28] T Tokuzawa, H Tsuchiya, T Tsujimura, M Emoto, H Nakanishi, S Inagaki, K Ida, H Yamada, A Ejiri, K Y Watanabe, et al 2018 Rev. Sci. Instrum. 89 10H118

[29] K Fujii, C Suzuki, and M Hasuo 2019 IEEE Trans. Plasma Sci. 47 3305-3314

[30] S Sakakibara, H Yamada, and LHD Experiment Group 2010 Fusion Sci. Tech. 58 471-481 